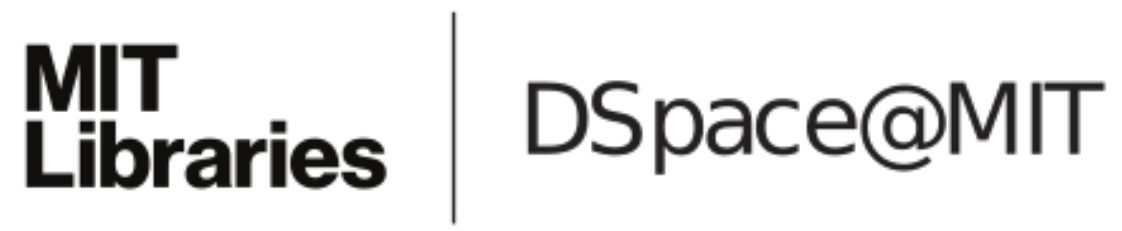

\author{
MIT Open Access Articles
}

Correlative dark-field and photoluminescence spectroscopy of individual plasmon-molecule hybrid nanostructures in a strong coupling regime

The MIT Faculty has made this article openly available. Please share how this access benefits you. Your story matters.

Citation: Wersäll, Martin, et al., "Correlative dark-field and photoluminescence spectroscopy of individual plasmon-molecule hybrid nanostructures in a strong coupling regime." ACS Photonics 6, 10 (Sept. 2019): p. 2570-76 doi 10.1021/acsphotonics.9b01079 (C2019 Author(s)

As Published: 10.1021/acsphotonics.9b01079

Publisher: American Chemical Society (ACS)

Persistent URL: https://hdl.handle.net/1721.1/125960

Version: Author's final manuscript: final author's manuscript post peer review, without publisher's formatting or copy editing

Terms of Use: Article is made available in accordance with the publisher's policy and may be subject to US copyright law. Please refer to the publisher's site for terms of use. 


\title{
Correlative dark-field and photoluminescence spectroscopy of individual plasmon-molecule hybrid nanostructures in strong coupling regime
}

\author{
Martin Wersäll ${ }^{1}$, Battulga Munkhbat ${ }^{1}$, Denis G. Baranov ${ }^{1}$, Felipe Herrera², Jianshu Cao ${ }^{3}$, \\ Tomasz J. Antosiewicz ${ }^{1,4}$, and Timur Shegai ${ }^{*}$ \\ ${ }^{1}$ Department of Physics, Chalmers University of Technology, 412 96, Göteborg, Sweden \\ ${ }^{2}$ Department of Physics, Universidad de Santiago de Chile, Avenida Ecuador 3943, Santiago, \\ Chile \\ ${ }^{3}$ Department of Chemistry, Massachusetts Institute of Technology, 77 Massachusetts Avenue, \\ Cambridge, Massachusetts 02139, USA \\ ${ }^{4}$ Faculty of Physics, University of Warsaw, Pasteura 5, 02-093 Warsaw, Poland \\ e-mail: timurs@chalmers.se
}

\begin{abstract}
Light-matter interactions play a crucial role in several prominent nanooptical phenomena, such as plasmon-mediated fluorescence, nanoscale lasing, and strong plasmon-exciton coupling. The latter holds promise for development of nanoscale nonlinear optical schemes and room temperature polaritonic lasers. In recent years, strong coupling in nanoscale plasmonexciton systems, also known as plasmon-exciton polaritons, have been thoroughly investigated using transmission, reflection and dark-field scattering spectroscopies. However, only a few recent studies performed experiments using photoluminescence spectroscopy on the individual hybrid nanostructure level. The latter is important for detailed understanding of intrinsic excited state dynamics in strongly coupled systems. Here, we use correlative dark-field scattering (DF) and photoluminescence (PL) measurements to study polaritonic states in individual silver nanoprisms surrounded by molecular J-aggregates. We investigate these systems under various experimental conditions, including temperatures in the range $\mathrm{T}=4-300 \mathrm{~K}$, laser excitation wavelengths at 532, 568, and $640 \mathrm{~nm}$, and broad range of plasmon-exciton detunings. Our findings indicate that lower energy peak in PL emission closely follows the lower polariton band observed in DF, while the higher energy PL peak follows the emission of uncoupled Jaggregate molecules and/or incoherent states. These observations further improve the understanding of excited state dynamics in strongly coupled plasmon-exciton systems.
\end{abstract}

KEYWORDS: Strong coupling; surface plasmon; plasmon-exciton coupling; photoluminescence; single nanoparticle spectroscopy; J-aggregates 
Strong coupling between collective electronic excitations, plasmons, in noble metal nanoparticles and optical absorption bands in organic chromophores have attracted significant research interest recently ${ }^{1,2}$. In this regime, the rate of coherent energy exchange between the plasmonic and molecular subparts exceeds their respective dissipation rates. To achieve sufficient coupling strength, a single plasmonic nanoparticle is often coupled to many chromophores, thereby forming coherent polaritonic states and a multitude of dark incoherent states. In the first approximation, such situation can be modelled by a single cavity mode coupled to $N>>1$ non-interacting emitters. This is known as the Tavis-Cummings model ${ }^{3}$. The diagonalization of Tavis-Cummings Hamiltonian results in two coherent bright polaritonic states - upper and lower polaritons, and $\mathrm{N}-1$ incoherent dark polaritonic states, whose photonic component is negligible. The existence of these bright and dark states, as well as the interplay between them, is crucial for relaxation processes in such hybrid systems ${ }^{4-8}$.

Following these concepts, many recent experiments have demonstrated strong coupling using high concentrations of organic dyes interacting with various optical resonators, such as propagating surface plasmons in thin metal films, Fabry-Pérot microcavities, and plasmonic nanoparticle arrays ${ }^{9-15}$. More recently experimental studies on single nanoparticle systems coupled to molecular excitons ${ }^{16-19}$, as well as excitons in transition metal dichalcogenides ${ }^{20-24}$ have been reported. Furthermore, several recent works claimed achieving strong coupling using individual quantum emitters, such as colloidal quantum dots ${ }^{25-27}$ and organic dyes $^{28}$.

Most plasmon-molecule studies listed above have relied on elastic spectroscopies, such as DF scattering, to quantify strong coupling. However, inelastic PL spectra must also be affected by strong coupling, as has been theoretically predicted earlier ${ }^{7,29,30}$. Recently several works have reported mode hybridization in PL signal from hybrid nanostructures, both on ensemble and single nanoparticle level ${ }^{8,26,27,31}$. Detailed correlative studies are, however, relatively sparse.

Here, we perform a correlative dark-field and photoluminescence spectroscopy study, using three different excitation wavelengths $-532,568$ and $640 \mathrm{~nm}$, at different temperatures ( $\mathrm{T}=4-300 \mathrm{~K}$ ), and for various plasmon-exciton detunings. The temperature-dependent data reveal unusual spectroscopic behavior of polaritonic states, which allows assigning the lower energy PL emission peak to the lower polariton, whereas the higher energy emission peak to uncoupled molecules and/or incoherent states. Noteworthy, no PL emission at the upper polariton frequency was observed in this study even at $\mathrm{T}=4 \mathrm{~K}$.

\section{Results and Discussion}

System under study. Figure 1a shows the sketch of a hybrid nanostructure. We use thin $(\sim 10 \mathrm{~nm})$ single-crystalline silver nanoprisms surrounded by J-aggregated TDBC molecules. The silver nanoprisms are about $\sim 70 \mathrm{~nm}$ in side length, such that the localized surface plasmon resonance matches the J-aggregate absorption line $(\sim 588 \mathrm{~nm})$. These hybrid nanostructures were synthesized in an aqueous solution using a photo-induced growth method (see Methods). 

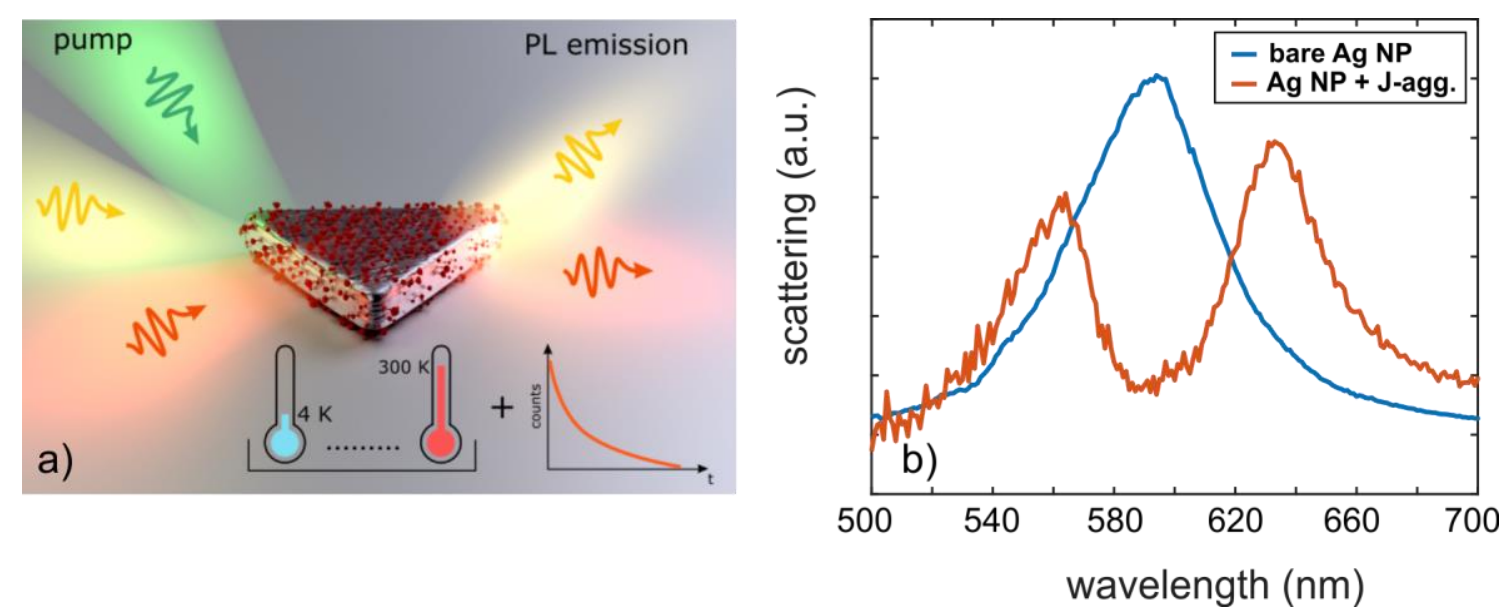

Figure 1. (a) Sketch of a nanoscale plasmon-exciton hybrid system - a single crystalline Ag nanoprism coated with J-aggregates of TDBC dye molecules, studied using PL and DF spectroscopy at different excitation lasers, temperatures ( $\mathrm{T}=4-300 \mathrm{~K}$ ), and plasmon-exciton detunings. (b) Comparison between a typical experimental DF scattering of an individual bare nanoprism (blue) and a nanoprism covered with J-aggregates (red). In the latter case a clear mode splitting of $\sim 250 \mathrm{meV}$ is observed.

Our hybrid nanostructures exhibit Rabi splitting in DF on the order of $\sim 200-250 \mathrm{meV}$, which exceeds both plasmon ( 150-200 meV) and exciton ( $\sim 50 \mathrm{meV})$ linewidths (see Fig. 1b). The single-crystalline nature and small dimensions of the silver nanoprisms result in relatively high quality factors for plasmonic nanoparticles Q 10-15, as well as subwavelength effective mode volumes, which makes them optimal for strong coupling. Most hybrid nanostructures in this study fulfill the criterion for the strong coupling regime, in agreement with our previous studies ${ }^{8,19,32}$.

PL with varying laser excitation wavelengths. PL spectra from three different single nanoparticles, each of which were excited using three different laser excitations (532, 568 and $640 \mathrm{~nm}$ ), together with the corresponding DF and scanning electron microscopy (SEM) images are shown in Figure 2. These measurements were conducted at $\mathrm{T}=77 \mathrm{~K}$ using an optical cryostat (see Methods). The nanoparticles are sorted left to right according to the increasing Rabi splitting to illustrate the PL and DF spectra differences between systems with varying coupling strengths. We additionally point out that under $532 \mathrm{~nm}$ excitation the PL spectra contain two resolved peaks, which we attribute to emission from incoherent dark states and/or uncoupled molecules (higher energy peak), and emission from the lower polariton (LP) state (lower energy peak). The arguments for this assignment will be provided further in the discussion. 

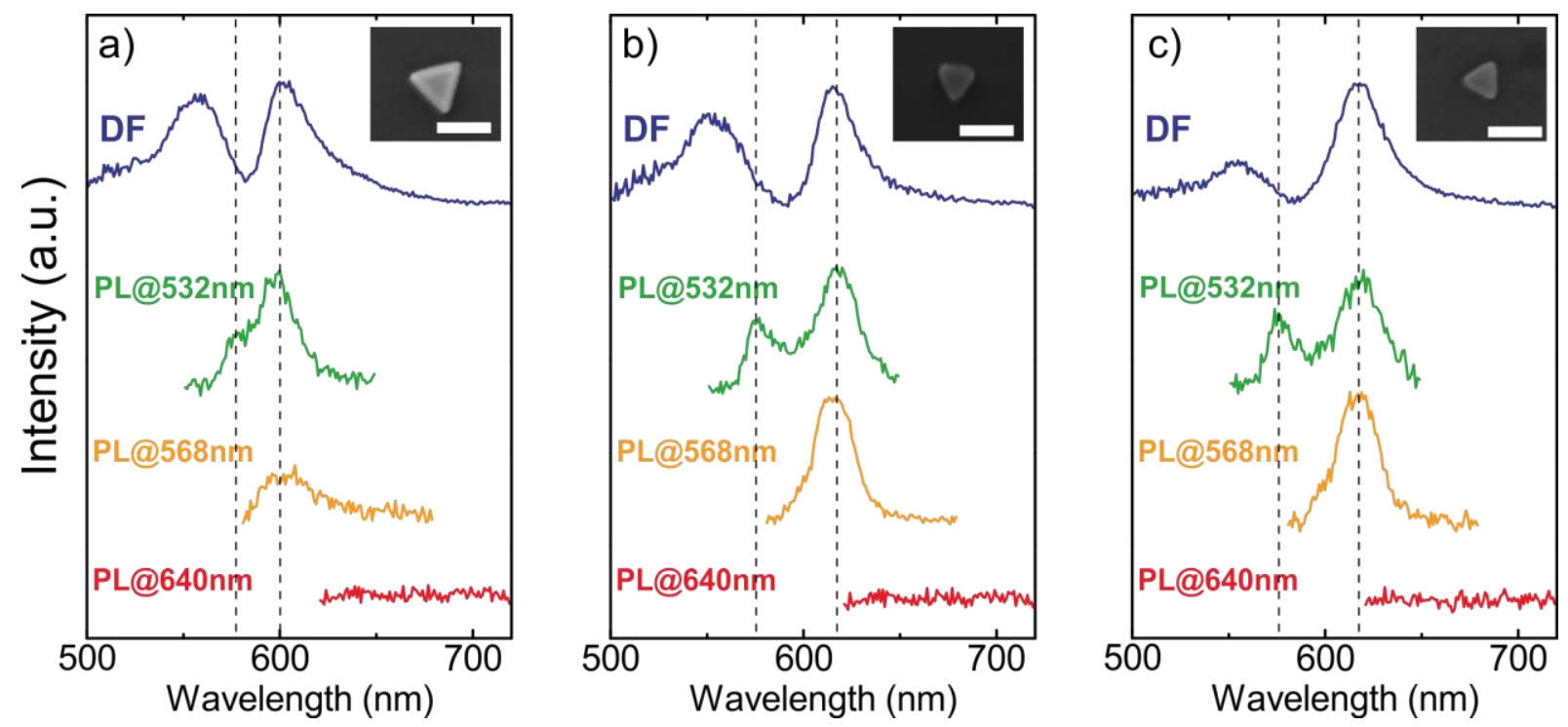

Figure 2. DF scattering (blue) and PL spectra of three different hybrid nanostructures excited by $532 \mathrm{~nm}$ (green), $568 \mathrm{~nm}$ (orange) and $640 \mathrm{~nm}$ (red) laser excitations. The particles in (a-c) are sorted left to right according to increasing vacuum Rabi splitting. The insets display SEM images of each corresponding hybrid nanostructure. The scale bars are $100 \mathrm{~nm}$. All measurements were conducted at $\mathrm{T}=77 \mathrm{~K}$ in an optical cryostat. Vertical dashed lines are guides for an eye.

The long-pass filters, which we employ to collect PL spectra for 568 and $640 \mathrm{~nm}$ laser excitations, allow transmitting wavelengths only above $580-590 \mathrm{~nm}$. It is in this spectral range where the emission from uncoupled molecules or incoherent states occurs. Hence, the high energy peaks would be absent in the PL spectra, even if the PL pathways would actually allow this kind of emission process to occur. Thus, upon $568 \mathrm{~nm}$ excitation, we can only detect the lower polariton emission in PL. At $640 \mathrm{~nm}$ excitation, however, almost no PL signal was detected for any of the studied nanoparticles. This could be due to low probability of populating long-lived incoherent dark states under our experimental conditions. In particular, the Rabi splitting in our systems is quite high, around $200-250 \mathrm{meV}$, while the inhomogeneous width of J-aggregates is much narrower, which makes it unlikely to populate the long-lived states upon resonant excitation of LP state. To further explain these observations, we refer the reader to the Supporting Information (Appendix: the model and comparison to experiment) for the detailed discussion about the mechanism of PL from strongly coupled systems.

Note that the lower energy PL emission peaks are almost spectrally overlapped with the corresponding lower polariton peaks in DF, which implies that the lower energy PL peaks correspond to the lower polariton emission. This observation is in line with previous experimental results ${ }^{8}$, as well as with theoretical models of plasmon-exciton hybrid nanostructures in the strong coupling regime ${ }^{7,29,30}$.

Furthermore, in Figure 3 we show correlation between all nanostructures that we measured in this study, sorted according to the increasing energy of the LP peak in DF. These experiments were conducted at $\mathrm{T}=77 \mathrm{~K}$. The data contains higher energy and lower energy peaks extracted from DF spectra, as well as the corresponding peaks extracted from PL data 
obtained using 532 and $568 \mathrm{~nm}$ excitation (at $640 \mathrm{~nm}$ excitation, no emission was detected). For $532 \mathrm{~nm}$ excitation, we provide both the higher energy and lower energy PL peaks data.

We notice several important details upon closer inspection of Figure 3. First, the upper polariton (UP) peak in DF fluctuates somewhat for different nanoparticles (see blue squares in Figure 3). This is likely due to variation in Rabi splitting of different nanostructures, which arises due to variation in their geometrical parameters, as well as the number and possibly orientation of J-aggregates around those structures. Despite these significant fluctuations in UP DF data, however, the higher energy PL peak (see orange circles in Figure 3) shows nearly no fluctuations and correspondingly no correlation with the UP peak in DF. This observation, together with the fact that high energy PL peak is significantly to the red from the UP DF, allows ruling out the upper polaritonic origin of the higher energy PL peak.

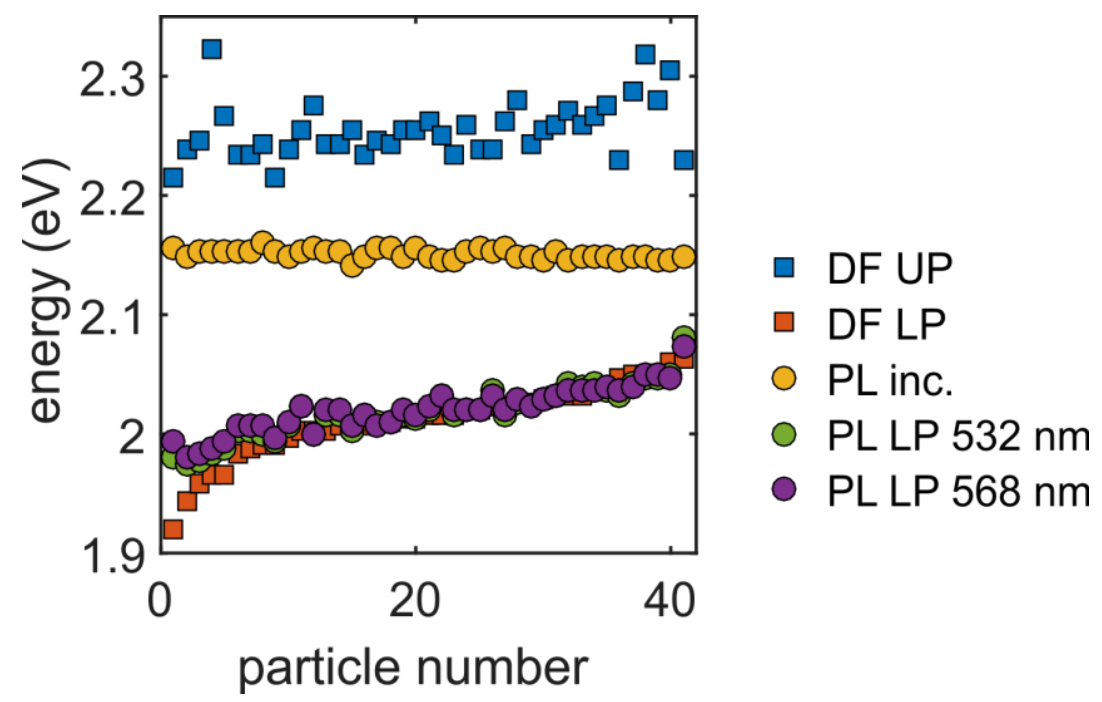

Figure 3. Correlative DF and PL (collected using 532 and $568 \mathrm{~nm}$ laser excitations) spectroscopy data, presented in terms of corresponding peak positions versus the nanoparticle number. The nanoparticles are arranged according to the increasing LP energy observed in DF. It is important to note that DF UP (blue squares) signal fluctuates, whereas higher energy PL peaks (orange circles) do not fluctuate with the nanoparticle number. This observation rules out the upper polariton origin of the higher energy PL peaks. At the same time the lower polariton DF and PL data at both excitation wavelengths are almost overlapping, confirming the polaritonic origin of lower energy PL peaks. All measurements were conducted at $\mathrm{T}=77 \mathrm{~K}$ in an optical cryostat.

Second, the LP in DF and lower energy PL peaks at both $532 \mathrm{~nm}$ and $568 \mathrm{~nm}$ laser excitations are almost overlapping with each other, which points to the lower polariton origin of the PL emission in this case, in agreement with data shown in Figure 2. The only deviation from this behavior is observed for particles with relatively low energy LPs (below $2.0 \mathrm{eVs}$ ). This could possibly be due to phonon-assisted relaxation pathway of PL in strongly coupled systems ${ }^{4-6,8}$. For strongly red-detuned particles, such relaxation requires large energy phonons. Those in turn might not be available to the system, as the phonon spectrum of J-aggregates has a high energy cutoff at around $1615 \mathrm{~cm}^{-1}$, which corresponds to $\sim 200 \mathrm{meV}$ (the high-frequency $\mathrm{C}-\mathrm{H}$ vibrations occur at much higher frequencies of around $\left.3500 \mathrm{~cm}^{-1}\right)^{17,33}$. This suggests that LP energies in DF and PL may start deviating from each other for nanoparticles with energy gap between the incoherent states (at around $2.15 \mathrm{eV}$ ) and LP exceeding the high energy cutoff 
in the J-aggregate phonon spectrum $\sim 200 \mathrm{meV}$, i.e. for LP DF energies below $1.95 \mathrm{eV}$. This agrees well with observations in Figure 3, thus supporting the validity of phonon-assisted relaxation in this case.

Role of plasmon-exciton detuning. Plasmon-exciton detuning plays an important role in the strong coupling, as it determines the composition of hybrid states. We will now study how PL depends on detuning between plasmon and exciton resonances in a hybrid system, $\delta=\omega_{p l}-$ $\omega_{0}$. Since the energy difference between the dark incoherent states (also called exciton reservoir) and upper/lower coherent polaritonic states is equidistant only in the case of zero detuning, it is important to study how the relaxation processes alter when this condition is not met. For a strongly blue-detuned situation, the dark states are closer to the lower polariton state, whereas in the strongly red-detuned case the dark states are closer to the upper polariton. The plasmon-exciton detuning thus plays an important role in the relaxation pathways of a hybrid system, especially in light of the phonon-assisted relaxation processes described in the previous section (also see SI Appendix: the model and comparison to experiment). Indeed, plasmon-exciton detuning has been previously shown to substantially affect the photo-oxidation rate of J-aggregates in the strong coupling regime ${ }^{32}$. Similar observations were also recently made in more extended organic microcavities ${ }^{34}$.

Blue Detuning
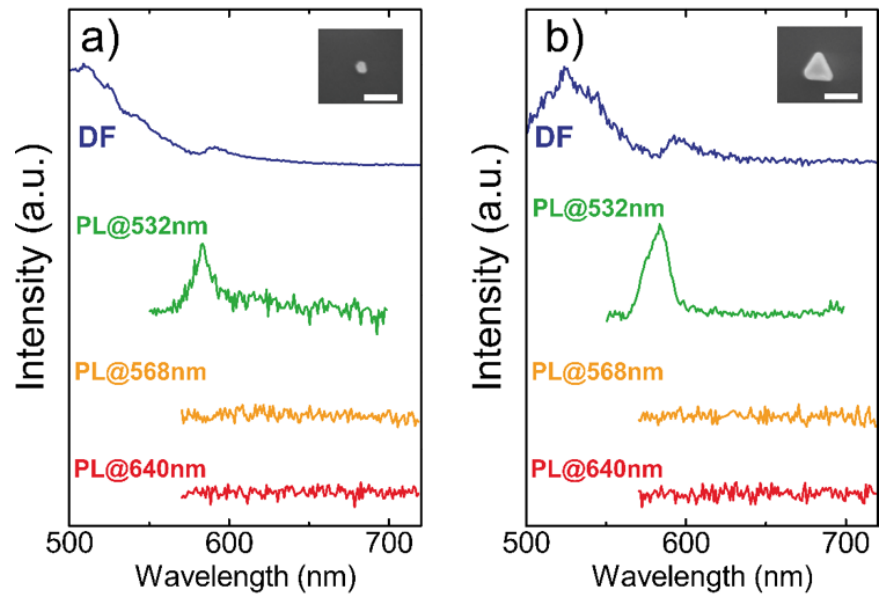

Red Detuning
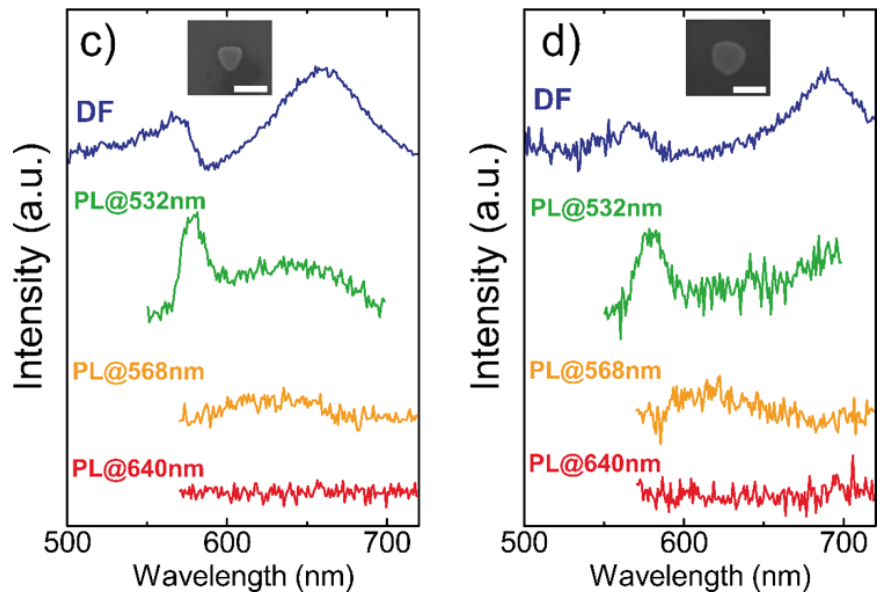
Figure 4. (a-b) Highly blue-detuned DF spectra together with the corresponding PL spectra obtained using 532, 568 and $640 \mathrm{~nm}$ laser excitations. (c-d) Highly red-detuned DF spectra together with the corresponding PL spectra obtained using 532, 568 and $640 \mathrm{~nm}$ laser excitations. Insets show corresponding SEM images. Scale bars are $100 \mathrm{~nm}$. All measurements were conducted at $\mathrm{T}=77 \mathrm{~K}$.

In Figure 4, experimental DF together with PL from highly blue-detuned (a-b), as well as highly red-detuned (c-d) plasmon-exciton hybrid systems are shown. The key observations for blue-detuned nanoparticles are as follows. First, at $532 \mathrm{~nm}$ excitation the emission from incoherent states is seen; however, at both 568 and $640 \mathrm{~nm}$ excitations, no detectable PL emission is observed. This is likely because the long-pass filters used for 568 and $640 \mathrm{~nm}$ excitations do not allow the incoherent molecular emission through. Moreover, the LP emission in this case is too close to the exciton reservoir, and thus cannot be efficiently populated by the phonon-assisted process.

For red-detuned particles, both incoherent states and LP emission is observed, similar to the close-to-zero detuning data in Figure 2. The only notable difference with respect to the latter is that the LP emission is relatively broad and shifted to the blue of the respective DF peak (in agreement with the low energy LP nanoparticles in Figure 3). These observations are in line with the Agranovich and Litinskaya formalism ${ }^{4-6}$, which is described in more details in the Appendix (see Supporting Information). In brief, this analytical formalism is based on the energy-conserving Fermi's golden rule and treats the polariton emission as a composition of phonon-assisted relaxation and radiative processes.

Finally, we note that in addition to the static PL spectra at $\mathrm{T}=77 \mathrm{~K}$, shown in Figures 2-4, we also performed lifetime measurements of the strongly coupled plasmon-molecule hybrids and compared them to bare J-aggregates. Unfortunately, the time resolution of our time correlated single photon counting modules (TCSPC) did not allow to resolve the ultrafast dynamics in the J-aggregates beyond the instrument response function (IRF). These results are therefore inconclusive, nevertheless we report them in the supporting information for the convenience of the readers (see SI Figure S3).

Temperature dependence of hybrid states spectroscopy. To further understand the relaxation processes of the hybrid structures in this study, we aim to investigate how both DF and PL spectra alter upon changes from cryogenic to room temperatures. The main reason for this is that inelastic relaxations within hybrid systems involve phonon-assisted energy dissipation ${ }^{4-7}$, 29, 30,35-37 and exciton-phonon interactions are known to depend on temperature ${ }^{38}$. It is therefore natural to investigate the presence of any temperature effects.

The spectral changes when the temperature is raised from $\mathrm{T}=4 \mathrm{~K}$ to $300 \mathrm{~K}$, both in $\mathrm{DF}$ and PL, are shown in Figure 5. Note that all spectra originate from the very same hybrid system at different temperatures. We also note that no photo-degradation of the sample was observed under the experimental conditions in the whole temperature range, allowing direct comparison between the different temperature-dependent PL spectra. The nanoparticle in this case was excited by a $532 \mathrm{~nm}$ laser. The inset displays the corresponding SEM image. 
Probably the most interesting and peculiar spectroscopic features of the strongly coupled system are observed in the $\mathrm{T}=150-300 \mathrm{~K}$ range, while below $\mathrm{T}=150 \mathrm{~K}$ the spectra are relatively temperature-independent. In particular, a substantial non-monotonic red shift of both polaritonic branches in DF is observed at $\mathrm{T}=200-250 \mathrm{~K}$, whereas upon further cooling, both peaks shift to the blue. This non-monotonic temperature behavior correlates well only with the lower energy PL peak, whereas the higher energy PL peak does not follow this path. Instead, it follows the temperature behavior of the uncoupled J-aggregates (see Figure S1). Noteworthy, both uncoupled plasmons and uncoupled J-aggregates show relatively weak and monotonic temperature-dependent spectra (see Figure S1), which is in sharp contrast to the strongly coupled systems. These temperature-dependent measurements therefore allow to conclude that the lower energy PL peaks are indeed due to the lower polariton, in full agreement with the data recorded at fixed T=77 K in Fig. 2-4. Correspondingly, the higher energy PL peak is not due to the upper polariton. Instead, the higher energy PL peak is due to emission from incoherent states and/or uncoupled molecules.

Additionally, it is noteworthy that the spectroscopic peculiarity, in all studied cases, seems to appear around the Debye temperature of silver $\left(T_{\Theta} \approx 221 \mathrm{~K}\right.$, see Appendix for more details). Such temperature dependence is reproducible and further examples may be found in Figure S4. Noteworthy, no PL emission at the upper polariton frequency was observed in this study even at $\mathrm{T}=4 \mathrm{~K}$. This is likely due to ultrafast relaxation into the long-lived dark states, with a subsequent decay into the lower polariton.
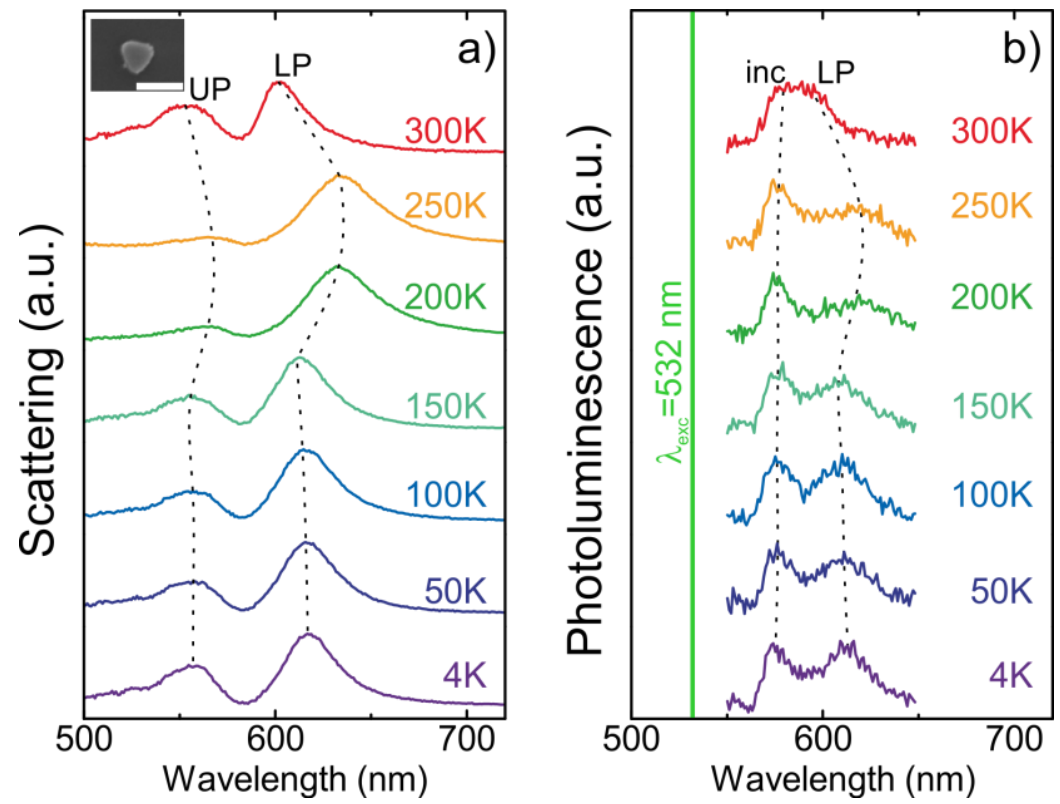

Figure 5. (a) Temperature-dependent DF spectra of a single hybrid nanostructure measured at $\mathrm{T}=4-300 \mathrm{~K}$. The inset shows SEM image of the corresponding nanoparticle. Scale bar is $100 \mathrm{~nm}$. (b) PL spectra recorded under $532 \mathrm{~nm}$ laser excitation for the same hybrid nanostructure and at the same temperatures as in (a). Dashed lines are guides for an eye. Note the substantial red shift of both UP and LP peaks in DF observed at T=200-250 K, whereas the corresponding temperature-dependent peculiarity in PL spectra is observed only for the lower energy PL peaks. The higher energy PL peaks instead follow the temperature dependence of uncoupled J-aggregates. 
The non-monotonic temperature dependence of the lower polariton PL peak in Figure $5 \mathrm{~b}$ can be understood using a polaron master equation approach ${ }^{39}$. By assuming that electronic excitations in individual aggregates are coupled to a harmonic oscillator bath with known spectral density $J(\omega)$, the lower polariton energy relative to the bare electronic resonance can be written as $E_{\mathrm{LP}}(T)=-\sqrt{N}\left(\Omega_{0} / 2\right) e^{-\Lambda(T)}-N \Omega_{0}^{2} C(T)$, where $\Omega_{0}$ is the bare Rabi frequency of an individual aggregate at zero temperature, and $N$ is the number of aggregates. The first term is the usual collective Rabi splitting ( $\sqrt{N}$-scaling), modulated by a decreasing exponential factor with thermal exponent $\Lambda(T)$. The term that scales linearly with $N$ corresponds to the bath-induced collective Lamb shift. At low temperatures, both $\Lambda(T)$ and $C(T)$ scale as $a+b T^{2}$ with temperature ( $a$ and $b$ being specific for $\Lambda$ and $C$ ). For $\mathrm{T} \leq 100 \mathrm{~K}$, both $\Lambda(T)$ and $C(T)$ are thus effectively temperature-independent, in agreement with Figure 5. Near $T \approx 150 \mathrm{~K}$, the Lamb shift term dominates the Rabi splitting due its stronger number scaling $(N$ vs $\sqrt{N})$. The lower polariton thus predominantly shifts to longer wavelengths with increasing temperature as $T^{2}$, up to a crossover temperature $T_{c}$. For temperatures beyond $T_{c}$, the lower polariton peaks tends toward the bare electronic resonance. This occurs because the functions $C(T)$ and $e^{-\Lambda(T)}$ both decay rapidly with temperature in this regime. The open quantum system model that describes the results in Figure 5 is summarized in the Supporting Information Section S5. Further theoretical details will be provided elsewhere ${ }^{40}$.

Conclusions. To conclude, we have performed a thorough correlative study between darkfield and photoluminescence spectroscopy of individual plasmon-molecule hybrid nanostructures in the strong coupling regime. The experiments were performed on individual silver nanoprisms surrounded by TDBC J-aggregates in a wide temperature range ( $\mathrm{T}=4$ $300 \mathrm{~K})$, under various laser excitations $(532,568$, and $640 \mathrm{~nm})$, and as a function of plasmonexciton detuning. To ensure single particle performance, all optical data were accompanied with SEM imaging. To avoid any photo-degradation of J-aggregates, all experiments were conducted using an optical cryostat.

Photoluminescence spectra for 532, 568, and $640 \mathrm{~nm}$ laser excitations show two, one or no distinct emission peaks, correspondingly. In case of $532 \mathrm{~nm}$ and $568 \mathrm{~nm}$ excitations, the lower energy PL peaks overlap with the corresponding LP DF peaks. Photoluminescence data from hybrids with blue and red plasmon-molecule detuning was studied. The presence of the lower polariton in PL is negligible in the blue-detuned cases, whereas in the red-detuned cases the lower polariton state is present, however, it appears broader and blue-shifted in comparison to the resonant case. Finally, temperature-dependent measurements show distinct red shifts in both polaritonic peaks occurring at $\mathrm{T}=200-250 \mathrm{~K}$ in dark-field spectra, which appear around the Debye temperature of silver $\left(T_{\Theta} \approx 221 \mathrm{~K}\right)$. Such behavior can be explained by the polaron master equation approach. The corresponding PL spectra show the temperature-induced peculiarity only in the lower energy emission peak. The higher energy peak instead follows the temperature behavior of uncoupled molecules, which is almost temperature-independent. These experiments independently point towards lower polaritonic nature of the lower energy peak in 
PL, while the higher energy peak in PL is likely due to uncoupled molecules and/or incoherent states. These observations are in line with the previously reported theoretical models ${ }^{4-7,29,30}$.

Thus, our experimental findings improve the fundamental understanding of relaxation processes within plasmon-exciton hybrid systems. Since there has been a limited number of experimental studies on this matter ${ }^{8,26,27,31}$, it is important to further broaden the knowledge about the relaxation processes occurring in hybrid systems to facilitate development of future applications, such as stable nanoparticle hybrids ${ }^{32}$ and room temperature single nanoparticle polaritonic lasers.

\section{Methods}

Synthesis of silver nanoprisms. In brief, a 3-5 nm silver seed nanoparticles solution was prepared by adding $1 \mathrm{~mL}$ of $30 \mathrm{mM}$ trisodium citrate and $0.5 \mathrm{~mL}$ of $20 \mathrm{mM}$ silver nitrate $\left(\mathrm{AgNO}_{3}\right)$ solutions to $95 \mathrm{~mL}$ of an ice-cold ultra-pure distilled water. The solution was kept bubbling with $\mathrm{N}_{2}$ under vigorous stirring in an ice-bath for an additional $60 \mathrm{~min}$ in the dark. Thereafter, $1 \mathrm{~mL}$ of ice-cold $50 \mathrm{mM} \mathrm{NaBH}_{4}$ was added into the growth solution, at which point the color of the solution turns pale-yellow. Then $100 \mu \mathrm{L}$ of $50 \mathrm{mM} \mathrm{NaBH}_{4}$ was added subsequently to the solution. This procedure was repeated 3 more times with 2 min break in between. The ice-cold and freshly prepared mixture of $1 \mathrm{~mL}$ of $5 \mathrm{mM} \mathrm{BSPP}$ and $1 \mathrm{~mL}$ of $50 \mathrm{mM} \mathrm{NaBH}_{4}$ was added dropwise into the growth solution for seed nanoparticles. The solution was kept for 5 hours in an ice bath under gentle stirring and completed with aging overnight in an ice bath under dark condition. For the photo-induced growth of silver nanoparticles, $10 \mathrm{~mL}$ of the aged seed solution with $\mathrm{pH}=9.5$ was irradiated with a $532 \mathrm{~nm}$ continuous-wave (CW) laser. The reaction was allowed to proceed for 24 hours, at which point the silver nanoparticles were washed two times by centrifugation at $3000 \mathrm{rcf}$ for $5 \mathrm{~min}$, where upon the supernatant was removed, and the remaining particles were re-dispersed in aqueous solution containing $0.3 \mathrm{mM}$ of trisodium citrate.

Synthesis of the hybrid nanostructures. The hybrid nanostructures utilized in this study, consist of self-assembled J-aggregates of TDBC dye molecules (5,6-Dichloro-2- [[5,6-dichloro1-ethyl-3- (4-sulfobutyl)-benzimidazol-2-ylidene]-propenyl]-1-ethyl- 3-(4-sulfobutyl) benzimidazolium hydroxide, inner salt, sodium salt) on individual Ag nanoprisms, where the freshly synthesized $\mathrm{Ag}$ nanoparticles $(1 \mathrm{~mL})$ were finally embedded in a molecular shells by mixing with $1 \mathrm{~mL}$ of the aqueous solution containing $0.1 \mathrm{mM}$ TDBC dye molecules and $1 \mathrm{mM}$ of $\mathrm{KBr}$ under gentle stirring for $15 \mathrm{~min}$. The resulting colloidal solution was washed once by centrifugation (3000 rcf for $5 \mathrm{~min}$ ). The supernatant was removed and the remaining nanostructures were re-dispersed in distilled water, and stored at $4^{\circ} \mathrm{C}$ in the dark until use.

Optical spectroscopy. The sample was prepared by drop casting $20 \mu \mathrm{L}$ of hybrid nanoparticles solution on a polylysine-functionalized opaque $\mathrm{Si} / \mathrm{SiO}_{2}$ substrate. After $2 \mathrm{~min}$, the solution was removed with a rapid extensive nitrogen flow. Exploiting a nano-patterned $\mathrm{Si} / \mathrm{SiO}_{2}$ substrate 
allows for spectroscopic and morphological correlation, as well as sufficient heat conductivity at cryogenic temperatures.

Bare J-aggregates sample was prepared from the solution containing $0.1 \mathrm{mM}$ TDBC dye molecules and $1 \mathrm{mM}$ of $\mathrm{KBr}$ with same procedure as for the hybrid nanoparticles. Dark-field scattering spectra were recorded using a reflective microscope (Nikon Y-IDP equipped with a $20 \times / \mathrm{NA}=0.4$ bright-field objective) with a hyper-spectral imaging technique based on a liquid crystal tunable filter. Both room temperature and cryogenic measurements were performed when the substrates were located inside a cryostat chamber (JANIS ST-500-UC), pumped to high vacuum $\left(\sim 10^{-5}\right.$ Torr $)$. The working distance of the dark-field scattering objectives, incorporated in the microscope, are too low to account for the distance between the lens and the substrate surface when the sample is places inside the cryostat chamber. Then, in order to mimic the dark-field scattering an external laser driven white-light source was utilized (Energetiq LDLS) at a high incident angle. On order to detect only the scattered light the sine of angle of the incident light needs to exceed the NA $(\sim 0.6)$ of the collecting objective.

For photoluminescence spectroscopy, we used a picosecond pulsed super-continuum whitelight laser (WhiteLase Micro, Fianuim) together with a suitable set of excitation, dichroic, and emission filters for 532, 568 and $640 \mathrm{~nm}$ excitations (all from Semrock). The photoluminescence experiments were conducted in the same optical cryostat and at the same temperatures as the corresponding dark-field measurements.

Scanning Electron Microscopy. Scanning electron microscopy (SEM) in a high-vacuum environment, with an acceleration voltage of $5 \mathrm{kV}$ was utilized in order to image single silver nanoprisms. The prisms were located on top of an opaque silicon substrate with $55 \mathrm{~nm} \mathrm{SiO}_{2}$ layer on top, which ensured good enough electron conductivity to avoid any charging effects, which could cause distortion to the final image. The substrate was furthermore pre-patterned from electron-beam lithography with different quadrants labeled $A 1, A 2, \ldots, A 15$ to $01,02, \ldots, 015$, which enabled a straight forward navigation and hence also made it possible for a one-to-one correlation between optical scattering/PL data and SEM.

\section{Associated Content:}

The Supporting Information is available free of charge on the ACS Publications website.

Additional information, control experiments, lifetime measurements, and additional modelling (PDF) 


\section{References}

1. Törmä, P.; Barnes, W. L. Rep. Prog. Phys 2015, 78, (1), 013901.

2. Baranov, D. G.; Wersall, M.; Cuadra, J.; Antosiewicz, T. J.; Shegai, T. ACS Photonics 2017, 5, 24.

3. Tavis, M.; Cummings, F. W. Phys. Lett. A 1967, 25, 714-715.

4. Agranovich, V. M.; Litinskaia, M.; Lidzey, D. G. Phys. Rev. B 2003, 67, (8), 085311.

$5 . \quad$ Litinskaya, M.; Reineker, P.; Agranovich, V. M. Journal of Luminescence 2004, 110, (4), 364-372.

$6 . \quad$ Litinskaya, M.; Reineker, P.; Agranovich, V. M. Journal of Luminescence 2006, 119-120, 277-282.

$7 . \quad$ Herrera, F.; Spano, F. C. Physical Review Letters 2017, 118.

8. Wersäll, M.; Cuadra, J.; Antosiewicz, T. J.; Balci, S.; Shegai, T. Nano Lett. 2017, $17,(1), 551-558$.

9. Bellessa, J.; Bonnand, C.; Plenet, J. C.; Mugnier, J. Phys. Rev. Lett. 2004, 93, (3), 036404.

10. Dintinger, J.; Klein, S.; Bustos, F.; Barnes, W. L.; Ebbesen, T. W. Physical Review B 2005, 71, (3).

11. Vasa, P.; Wang, W.; Pomraenke, R.; Lammers, M.; Maiuri, M.; Manzoni, C.; Cerullo, G.; Lienau, C. Nature Photon. 2013, 7, (2), 128-132.

12. Schwartz, T.; Hutchison, J. A.; Genet, C.; Ebbesen, T. W. Phys. Rev. Lett. 2011, 106, (19), 196405.

13. $\quad$ Balci, S. Optics Letters 2013, 38, (21), 4498-4501.

14. Väkeväinen, A. I.; Moerland, R. J.; Rekola, H. T.; Eskelinen, A.-P.; Martikainen, J.-P.; Kim, D.-H.; Törmä, P. Nano Lett. 2014, 14, (4), 1721-1727.

15. Ramezani, M.; Halpin, A.; Fernandez-Dominguez, A. I.; Feist, J.; Rodriguez, S. R.-K.; Garcia-Vidal, F. J.; Rivas, J. G. Optica 2017, 4, 31-37.

16. Schlather, A. E.; Large, N.; Urban, A. S.; Nordlander, P.; Halas, N. J. Nano Lett. 2013, 13, (7), 3281-3286.

17. Zengin, G.; Johansson, G.; Johansson, P.; Antosiewicz, T. J.; Käll, M.; Shegai, T. Sci. Rep. 2013, 3, 3074.

18. Itoh, T.; Yamamoto, Y. S.; Tamaru, H.; Biju, V.; Wakida, S.-i.; Ozaki, Y. Phys. Rev. B 2014, 89, (19), 195436.

19. Zengin, G.; Wersäll, M.; Nilsson, S.; Antosiewicz, T. J.; Käll, M.; Shegai, T. Phys. Rev. Lett. 2015, 114, (15), 157401.

20. Wen, J.; Wang, H.; Wang, W.; Deng, Z.; Zhuang, C.; Zhang, Y.; Liu, F.; She, J.; Chen, J.; Chen, H.; Deng, S.; Xu, N. Nano Lett. 2017, 17, 4689.

21. Zheng, D.; Zhang, S.; Deng, Q.; Kang, M.; Nordlander, P.; Xu, H. Nano Lett. 2017, 17, 3809.

22. Kleemann, M.-E.; Chikkaraddy, R.; Alexeev, E. M.; Kos, D.; Carnegie, C.; Deacon, W.; de Pury, A. C.; Große, C.; de Nijs, B.; Mertens, J.; Tartakovskii, A. I.; Baumberg, J. J. Nature Communications 2017, 8, (1), 1296.

23. Cuadra, J.; Baranov, D. G.; Wersäll, M.; Verre, R.; Antosiewicz, T. J.; Shegai, T. Nano Lett. 2018, 18, 1777.

24. Stührenberg, M.; Munkhbat, B.; Baranov, D. G.; Cuadra, J.; Yankovich, A. B.; Antosiewicz, T. J.; Olsson, E.; Shegai, T. Nano Letters 2018, 18, (9), 5938-5945.

25. Santhosh, K.; Bitton, O.; Chuntonov, L.; Haran, G. Nat. Commun. 2016, 7, 11823.

26. Groß, H.; Hamm, J. M.; Tufarelli, T.; Hess, O.; Hecht, B. Science Advances 2018, 4, (3). 

2018, 9, (1), 4012.

28. $\quad$ Chikkaraddy, R.; de Nijs, B.; Benz, F.; Barrow, S. J.; Scherman, O. A.; Rosta, E.; Demetriadou, A.; Fox, P.; Hess, O.; Baumberg, J. J. Nature 2016, 535, (7610), 127-130.

29. del Pino, J.; Schröder, F. A. Y. N.; Chin, A. W.; Feist, J.; Garcia-Vidal, F. J. Physical Review Letters 2018, 121, (22), 227401.

30. $\quad$ Neuman, T.; Aizpurua, J. Optica 2018, 5, (10), 1247-1255.

31. Melnikau, D.; Esteban, R.; Savateeva, D.; Sánchez-Iglesias, A.; Grzelczak, M.; Schmidt, M. K.; Liz-Marzán, L. M.; Aizpurua, J.; Rakovich, Y. P. J. Phys. Chem. Lett. 2016, 7, (2), 354-362.

32. $\quad$ Munkhbat, B.; Wersäll, M.; Baranov, D. G.; Antosiewicz, T. J.; Shegai, T. Science Advances 2018, 4, (7), eaas9552.

33. Coles, D. M.; Meijer, A. J. H. M.; Tsoi, W. C.; Charlton, M. D. B.; Kim, J.-S.; Lidzey, D. G. The journal of physical chemistry A 2010, 114, (44), 11920-11927.

34. Peters, V. N.; Faruk, M. O.; Asane, J.; Alexander, R.; Peters, D. a. A.; Prayakarao, S.; Rout, S.; Noginov, M. A. Optica 2019, 6, (3), 318-325.

35. $\quad$ Michetti, P.; La Rocca, G. C. Physical Review B 2005, 71, (11), 115320.

36. $\quad$ Michetti, P.; La Rocca, G. C. Physical Review B 2008, 77, (19), 195301.

37. Coles, D. M.; Michetti, P.; Clark, C.; Tsoi, W. C.; Adawi, A. M.; Kim, J.-S.; Lidzey, D. G. Advanced Functional Materials 2011, 21, (19), 3691-3696.

38. $\quad$ Rudin, S.; Reinecke, T. L.; Segall, B. Physical Review B 1990, 42, (17), 1121811231.

39. $\quad$ Xu, D.; Cao, J. Frontiers of Physics 2016, 11, (4), 110308.

40. Herrera, F.; Wersäll, M.; Shegai, T.; Cao, J. 2019. in preparation 